\title{
Smart Charging, Vehicle-to-Grid, and Reactive Power Support from Electric Vehicles in Distribution Grids: A Performance Comparison
}

\author{
Biswarup Mukherjee \\ MINES ParisTech - PSL University \\ Centre PERSEE \\ Sophia Antipolis, France \\ biswarup.mukherjee@mines-paristech.fr
}

\author{
Georges Kariniotakis \\ MINES ParisTech - PSL University \\ Centre PERSEE \\ Sophia Antipolis, France \\ georges.kariniotakis@mines-paristech.fr
}

\author{
Fabrizio Sossan \\ MINES ParisTech - PSL University \\ Centre PERSEE \\ Sophia Antipolis, France \\ fabrizio.sossan@mines-paristech.fr
}

\begin{abstract}
This paper compares different charging strategies for electric vehicles (EVs) and mechanisms to support local distribution grids. First, a general scheduling problem for EVs based on convex optimization and linearized power grid models is presented. Then, it is shown how it can be adapted to model different charging strategies. These include: i) uncoordinated charging, where EVs maximize a local utility function regardless of grid constraints; ii) smart charging, where a charge schedule of all EVs is determined by maximizing their utility function subject to grid constraints; iii) vehicle-to-grid, where bidirectional power from the EVs is allowed; and iv) reactive power support, where 2- and 4-quadrant EV chargers can provide reactive power. The performance of these strategies are investigated considering the CIGRE benchmark system for medium-voltage distribution grids. It shows that, in the proposed scenario, smart charging with reactive power support is conducive to the shortest global recharging time.
\end{abstract}

Index Terms-Electric vehicles, Distribution grids, Uncoordinated charging, Smart charging, Vehicle-to-grid, Optimal scheduling.

\section{INTRODUCTION}

The transport sector is responsible for over one-third of the global greenhouse gas emissions and 27\% within Europe in 2017 [1]. Electric vehicles (EVs) will play an important role in reducing emissions of the road transport sector as well as dependency on fossil fuels [2], [3]. The increasing adoption of EVs requires efficient ways to manage their charge. Indeed, the simultaneous charging of a large population of EVs results in a significant increase of the electrical demand and may cause violations of distribution grids' operational constraints, possibly requiring expensive grid reinforcements to distribution system operators (DSOs), as for example highlighted in [4] for distribution grid transformers. Smart charging has been widely proposed in the existing literature to cope with the issues of uncoordinated charging, helping DSOs with congestion management (e.g., [3], [5]). Smart charging

This project has received funding in the framework of the joint programming initiative ERA-Net Smart Energy systems' focus initiative Integrated, Regional Energy Systems, with support from the European Union's Horizon 2020 research and innovation programme under grant agreement No 775970, in the context of the EVA project. consists in scheduling the charging process of EVs to avoid grid congestions. Vehicle-to-grid (V2G) with bi-directional chargers is one step further and refers to allowing EVs to discharge their batteries and inject power into the grid to support operations of both distribution and transmission grids (e.g., [6], [7]). Both unidirectional and bidirectional chargers can provide reactive power to the grid, which can be used to support local voltage regulation, e.g., [8], [9].

This paper investigates the performance of different EV charging strategies (uncoordinated, smart charging, $\mathrm{V} 2 \mathrm{G}$, and reactive power support) considering a medium-voltage (MV) grid and their impact on the recharging times. All these scheduling problems are derived from a baseline convex optimization problem that embeds linearized grid constraints from the existing literature [10]. The performance comparison of these strategies in a common setting and a unified algorithmic framework to accommodate multiple scheduling problems stand as the main contributions of this paper. The rest of this paper is organized as follows. Section II states the problem and introduces the models. Section III and IV describes the methods. Section V and VI present the case study and the results, and Section VII concludes the paper.

\section{Problem Statement AND MOdels}

\section{A. Problem statement}

We consider a distribution grid (e.g., Fig. 1) with loads (including EV chargers), and possibly distributed generation. The simultaneous charging of many EVs might cause violations of its operational constraints. These constraints include ensuring suitable nodal voltage magnitudes, line currents below cable ampacities, and power flow at the substation below the transformer rating. This paper aims to compare different charging strategies for EVs and evaluate how they impact grid constraints and recharging times. The considered recharging strategies are: uncoordinated charging (EVs recharge as soon as they are plugged in), smart charging (charge is scheduled to ensure that grid constraints are respected), V2G (as smart charging, but EVs are allowed to discharge to support the grid, if necessary), and reactive power support (as smart 
charging, but chargers can adjust reactive power injections). Grid constraints are modelled with a linearized grid model, as described next.

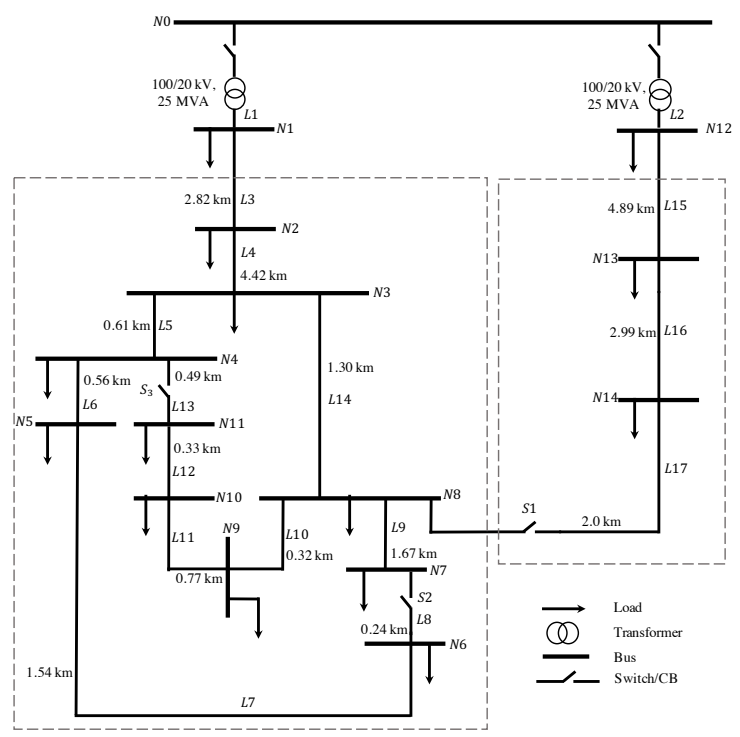

Fig. 1. Topology of the CIGRE European MV distribution network benchmark for residential system [11].

\section{B. Power grid model}

Let $n=1, \ldots, N$, denote the index of the node of the network, where $N$ is the total number of nodes. The index $t=1, \ldots, T$ refers to the time interval, with $T$ total samples. The time horizon $1, \ldots, T$ refers to the recharging horizon for the EVs. In the rest of this paper, we will refer to overnight charging, although the formulation is general and can be applied to other cases. Time dynamics need to be considered to model both time-variant power demand, and because the EVs' charging process is inherently time dependant due to the state-of-charge constraints of the EVs. The active and reactive power nodal injections at node $n$ and time interval $t$ is:

$$
\begin{aligned}
& P_{t n}=P_{t n}^{(\text {net })}+P_{t n}^{(\mathrm{EV} \text { nodal })} \\
& Q_{t n}=Q_{t n}^{(\mathrm{net})}+Q_{t n}^{(\mathrm{EV} \text { nodal })}
\end{aligned}
$$

for all nodes of the grid, where $P_{t n}^{(\text {net })}$ is the net demand, i.e., power demand minus local renewable generation and $P_{t n}^{(\mathrm{EV} \text { nodal })}$ is the total power demand of EVs. The former is an input of the problem, whereas the latter depends on the charging policy and is modelled as described in section II C.

Voltage levels at the nodes of the grid and the current values in the lines (voltage magnitudes, and line current magnitudes) depend on the grid topology, cables' parameters, voltage at the slack node, and nodal injections. They are modelled with load flow equations, that we generically denote in the following with functions $f_{n}$ for the voltage magnitudes and $h_{l}$ for line current magnitudes:

$$
\begin{aligned}
& v_{t n}=f_{n}\left(P_{t 1}, \ldots, P_{t N}, Q_{t 1}, \ldots, Q_{t N}, v_{0}, Y\right) \\
& i_{t l}=h_{l}\left(P_{t 1}, \ldots, P_{t N}, Q_{t 1}, \ldots, Q_{t N}, v_{0}, Y\right),
\end{aligned}
$$

where $Y$ is the admittance matrix of the grid (built using the grid topology information and cable parameters), and $v_{0}$ is the voltage at the slack bus.

Load flow equations are nonlinear and their inclusion in optimization problems determines nonconvex formulations. To increase tractability, we linearize (2) and (3) using the method proposed in [10] based on sensitivity coefficients, whose performance has been investigated in [12], and as done in [13].

\section{Modelling the charging of EVs}

Let $v=1, \ldots, V$ denote the index of a vehicle, where $V$ is the total number of vehicles. The state-of-charge of EV $v$ is modelled as the following function:

$$
\begin{aligned}
\operatorname{SOC}_{t+1, v} & \left(P_{1 v}^{\mathrm{EV}}, \ldots, P_{t v}^{\mathrm{EV}}\right)=\mathrm{SOC}_{0 v}+ \\
& +\sum_{\tau=1}^{t}\left(\eta_{v}\left[P_{\tau v}^{\mathrm{EV}}\right]^{+}-\frac{1}{\eta_{v}}\left[P_{\tau v}^{\mathrm{EV}}\right]^{-}\right) \frac{T_{s}}{E_{v}}
\end{aligned}
$$

where $\mathrm{SOC}_{0 v}$ is the initial $\mathrm{SOC}, P_{t v}^{\mathrm{EV}}$ is the EV charging power, operators $[\cdot]^{+}$and $[\cdot]^{-}$denote respectively the positive and negative arguments of the charging power $\left({ }^{1}\right), T_{s}$ is the sampling time (in hours), $E_{v}$ is the nominal energy capacity of the EV's battery in $\mathrm{kWh}$ ), and $\eta_{v}$ is the charging/discharging efficiency (possibly different across the population according to the charger model and battery status).

The charging power of vehicle $v$ is limited by the rated power of the converter, denoted by $\bar{S}_{v}^{\mathrm{EV}}$ (in kVA). We assume the capability of the charger is independent from the voltage of the AC grid and of the DC bus. The apparent power limit of the charger can be expressed as:

$$
\left(P_{t v}^{\mathrm{EV}}\right)^{2}+\left(Q_{t v}^{\mathrm{EV}}\right)^{2} \leq\left(\bar{S}_{v}^{\mathrm{EV}}\right)^{2}
$$

where $Q_{t v}^{\mathrm{EV}}$ is the charger's reactive power.

The link between the nodal injections of EVs, $P_{t n}^{(\text {EV nodal })}, Q_{t n}^{(\mathrm{EV} \text { nodal })}$ in (1), and the charging of the single vehicles $P_{t v}^{\mathrm{EV}}$ is given by the charging location where the EVs charge. The charging location of EV $v$ is encoded in the sequence of binary variables $b_{1 v}, b_{2 v}, \ldots, b_{N v}$, that contains a " 1 " in the node where the EV charges, and N-1 " 0 " at all other nodes. In this way, the nodal EV injections are given by:

$$
\begin{aligned}
P_{t n}^{(\mathrm{EV} \text { nodal })} & =\sum_{v=1}^{V} b_{n v} P_{t v}^{\mathrm{EV}} \\
Q_{t n}^{(\mathrm{EV} \text { nodal })} & =\sum_{v=1}^{V} b_{n v} Q_{t v}^{\mathrm{EV}}
\end{aligned}
$$

for all $n$. The $N \times V$ binary matrix $b_{n v}$ for all $n, v$ can be interpreted as a map reporting the recharging spots of all EVs. It is an input to the problem.

\footnotetext{
${ }^{1}$ In smart charging, the charging power is typically positive only, whereas, in $\mathrm{V} 2 \mathrm{G}$, it can be negative too when EVs are called to support the grid.
} 


\section{GENERAL FRAMEWORK}

This section describes a general formulation of the charge scheduling problem for the EVs that includes both V2G and reactive power support. The next section discusses how this formulation is modified to accommodate various scheduling strategies, which are finally compared in the results section.

The objective of this problem is determining a charging schedule for all EVs so that they can achieve a target stateof-charge while respecting grid constraints. The scheduling problem is formulated as a constrained optimization problem, whose constraints and cost function are described next. The EV chargers can possibly use reactive power and discharge the vehicles if this is conducive to improve the cost function.

For exemplifying the context, we consider a scenario where the EVs recharge overnight to achieve a target SOC level (e.g., a full recharge) necessary to meet the driving demand starting from an initial SOC value, smaller than the target SOC. The initial SOC is given by the state at which the EVs arrive at their parking (and charging) location. Both the initial SOC level and the target SOC level are inputs to the problem. They are respectively denoted by $\mathrm{SOC}_{0 v}$ and $\mathrm{SOC}_{v}^{\star}$.

\section{A. Cost function}

The cost function of the problem can be formulated to represent several operational objectives, such as minimizing the total charging time or minimizing the cost of electricity in a price-taking setting. Without losing generality, we choose to minimize the charging time; other cost function can be implemented too.

Let $\boldsymbol{p}=\left[P_{t v}^{\mathrm{EV}}\right.$ for all $\left.t, v\right]$ be a vector collecting all the charging power of all vehicles and time intervals, and $\boldsymbol{p}_{v}^{(t)}=$ $\left[P_{1 v}^{\mathrm{EV}}, \ldots, P_{t v}^{\mathrm{EV}}\right]$ a vector collecting the charging power from the initial time interval to $t$ for $\mathrm{EV} v$. We minimize the charging time by penalizing, for each vehicle, deviations of its state of charge from its target value, $\mathrm{SOC}_{v}^{\star}$. This reads as:

$$
J^{\prime}(\boldsymbol{p})=\sum_{t=1}^{T} \sum_{v=1}^{V}\left(\operatorname{SOC}_{t v}\left(\boldsymbol{p}_{v}^{(t-1)}\right)-\mathrm{SOC}_{v}^{*}\right)^{2},
$$

where $\operatorname{SOC}_{t v}(\cdot)$ is computed as in (4). The positive- and negative-part operators in (4) are non-linear and lead to a nonconvex formulation. Several linearizations are possible, e.g. splitting the charging power into mutually exclusive positive and negative terms (with binary variables, or penalization terms in the cost function) or embedding resistive losses in a virtual line, as in [14]. We opt for exclusive terms penalized in the cost function, as explained next, as it does not involve the use of binary variables.

\section{B. Linear model of the EVs state-of-charge}

Decision variable $P_{t v}^{\mathrm{EV}}$ is replaced with two new nonnegative variables:

$$
P_{t v}^{\mathrm{EV}}=P_{t v}^{\mathrm{EV}+}-P_{t v}^{\mathrm{EV}-} . \quad \forall v, t
$$

In this way, the state-of-charge can be defined as the linear expression:

$$
\begin{aligned}
& \operatorname{SOC}_{t+1, v}\left(P_{1 v}^{\mathrm{EV}+}, \ldots, P_{t v}^{\mathrm{EV}+}, P_{1 v}^{\mathrm{EV}-}, \ldots, P_{t v}^{\mathrm{EV}-}\right)= \\
&=\mathrm{SOC}_{0 v}+\sum_{\tau=1}^{t}\left(\eta_{v} P_{v \tau}^{\mathrm{EV}+}-\frac{1}{\eta_{v}} P_{v \tau}^{\mathrm{EV}-}\right) \frac{T_{s}}{E_{v}} .
\end{aligned}
$$

As a charger can either charge or discharge an EV, the two terms in (9) should be mutually exclusive. This is achieved by augmenting the cost function in (8) with a new term that penalizes $P^{\mathrm{EV}+}$ and $P^{\mathrm{EV}-}$. This augmented cost function reads as:

$$
J\left(\boldsymbol{p}^{+}, \boldsymbol{p}^{-}\right)=J^{\prime}\left(\boldsymbol{p}^{+}, \boldsymbol{p}^{-}\right)+k \sum_{t=1}^{T} \sum_{v=1}^{V}\left(P^{\mathrm{EV}+}+P^{\mathrm{EV}-}\right),
$$

where $\boldsymbol{p}^{+}=\left[P_{t v}^{\mathrm{EV}+}\right.$ for all $\left.t, v\right]$ and $\boldsymbol{p}^{-}=\left[P_{t v}^{\mathrm{EV}-}\right.$ for all $\left.t, v\right]$, the cost function $J^{\prime}\left(\boldsymbol{p}^{+}, \boldsymbol{p}^{-}\right)$follows from expressing SOC model (8) with model (10), and $k$ is a weight. As the second term of the cost function corresponds to the SOC's model relaxation and does not have a specific physical meaning, $k$ should be small to not significantly alter the original cost function. The impact of $k$ on the algorithm performance and on the original problem objective is small, as shown in the results.

\section{Problem constraints}

The problem constraints include: state-of-charge model (10) and limits (denoted by $\underline{\mathrm{SOC}}$ and $\overline{\mathrm{SOC}}$ ); EV charger rating in (5); EV charging-to-nodal injections models in (6)-(7), with $P_{t v}^{\mathrm{EV}}$ given by (9) and grid nodal injection models in (1a), (1b); linear grid constraints, i.e., voltage lower and upper limits $\underline{v}, \bar{v}$ at all nodes, cable ampacity $\bar{i}_{l}$ of all lines $l$, and transformer power rating $P_{t 0}^{2}+Q_{t 0}^{2} \leq \bar{S}_{0}^{2}$. The formulations are discussed next.

\section{Complete formulation}

The complete problem formulation is now presented. Problem's decision variables are $\boldsymbol{p}^{+}, \boldsymbol{p}^{-}$and the newly defined vector $\boldsymbol{q}=\left[Q_{t v}^{\mathrm{EV}}\right.$ for all $\left.t, v\right]$ that collects all the reactive power injections of all chargers over time. The problem reads as:

$$
\underset{\boldsymbol{p}^{+}, \boldsymbol{p}^{-} \in R_{+0}^{T \times V}, \boldsymbol{q} \in R^{T \times V}}{\arg \min } J\left(\boldsymbol{p}^{+}, \boldsymbol{p}^{-}\right)
$$

subject to:

$\begin{array}{ll}\mathrm{SOC} \text { model }(10) & \forall v, t \\ \underline{\mathrm{SOC}} \leq \mathrm{SOC}_{t v} \leq \overline{\mathrm{SOC}} & \forall t, v \\ \left(P_{t v}^{\mathrm{EV}+}-P_{t v}^{\mathrm{EV}-}\right)^{2}+\left(Q_{t v}^{\mathrm{EV}}\right)^{2} \leq\left(\bar{S}_{v}^{\mathrm{EV}}\right)^{2} & \forall t, v \\ \text { Nodal injections (6), (7), (9),(1a), (1b) } & \forall t, n, v \\ \text { Linear grid models (2)-(3) } & \forall t, n, l \\ \underline{v} \leq v_{t n} \leq \bar{v} & \forall t, n \\ \left|i_{t l}\right| \leq \bar{i}_{l} & \forall t, l \\ P_{t 0}^{2}+Q_{t 0}^{2} \leq \bar{S}_{0}^{2} & \forall t .\end{array}$




\section{VEHICLE-TO-GRID, SMART-CHARGING, AND UNCOORDINATED CHARGING}

\section{A. Vehicle-to-grid problem}

The optimization problem in (12) determines the charging schedule of all EVs so that the total charging time across the EV population is the smallest while subject to grid constraints. The decision variables are the charging power, the discharging power, and the reactive power. Although both the discharging power and the reactive power do not contribute to recharge the EVs directly (reactive power does not appear in (10)), their contribution might be useful to alleviate network congestions, ultimately allowing other EVs to recharge and improving the global charging time across the population. Problem (12) refers to the $\mathrm{V} 2 \mathrm{G}$ scheduling problem with reactive power support. The reactive power support can be excluded by either forcing $\boldsymbol{q}$ to zero with equality constraints or remove the associated decision variables from the problem and constraints.

\section{B. Smart charging problem}

The smart charging problem can be derived from (12) by either forcing $\boldsymbol{p}^{-}$to zero or removing $\boldsymbol{p}^{-}$from the decision variables. We opt for the second way. In this case, the cost function $J^{\prime}(\cdot)$ can be dropped in favor of $J(\cdot)$. Both the smart charging and V2G problems are centralized, in the sense that - in order to be solved - require collecting all the information into a single optimization problem. This is due to the grid constraints that depend on all nodal injections and are therefore coupling constraints.

\section{Uncoordinated charging}

In uncoordinated charging, each vehicles seek to minimize its charging time regardless of the grid constraints. This simplified problem can be derived from (12) and reads as:

$$
\underset{\boldsymbol{p}^{+}}{\arg \min } J^{\prime}\left(\boldsymbol{p}^{+}\right)
$$

subject to

$$
\begin{array}{ll}
\mathrm{SOC}_{t v}=\mathrm{SOC}_{t-1 v}+\eta_{v} \frac{T_{S}}{E_{v}} P_{t v}^{(\mathrm{EV})} & \text { for all } t \text { and } v \\
0 \leq \mathrm{SOC}_{t v} \leq 100 \%, & \text { for all } t \text { and } v \\
0 \leq P_{t v}^{\mathrm{EV}} \leq \bar{S}_{v}^{\mathrm{EV}} & \text { for all } t \text { and } v .
\end{array}
$$

This problem is separable because it has no coupling constraint. In other words, solving this problem is equivalent to solving $V$ optimization problems (one per vehicle) with local information only. The uncoordinated charging problem is used as benchmark scenario to evaluate the impact of grid constraints.

\section{CASE STUdy}

\section{A. Grid}

The CIGRE benchmark system for MV grids (European version) [11] is used as a case study (Fig. 1). The MV grid comprises low-voltage (LV) systems connected at the various nodes that are modelled in terms of their aggregated contributions. It is assumed that there are no grid constraint violations in the LV grids. The MV grid is modelled with a single-phase equivalent assuming a balanced grid with transposed conductors. The nodal nominal powers, the power factors, and the number of parked EVs at each node are reported in Table I. The time-varying active power demand is modeled with the load coincidence factor model defined in [11]. The reactive power demand is modeled as the product between the active power and the tangent of arc-cosine of the power factor in Table I. Line ampacities are according to the conductor diameter. Allowed voltage levels are within 1 per unit $\pm 3 \%$. Computing sensitivity coefficients for the linear grid models requires nominal active and reactive nodal injections to compute the linearization, for which we use the nodal power injections of the net demand from the CIGRE specifications. The EV input data are discussed next.

TABLE I

NODAL NOMINAL DEMAND PER NODE AND NUMBER OF EVS

\begin{tabular}{c|c|c|c}
\hline Node & $\begin{array}{c}\text { Apparent Power } \\
{[\mathrm{kVA}]}\end{array}$ & Power factor & Number of EVs \\
\hline 1 & $15^{\prime} 300$ & 0.98 & 0 \\
2 & 0 & 0 & 0 \\
3 & 285 & 0.97 & 68 \\
4 & 445 & 0.97 & 106 \\
5 & 750 & 0.97 & 178 \\
6 & 565 & 0.97 & 134 \\
7 & 0 & 0 & 0 \\
8 & 605 & 0.97 & 144 \\
9 & 0 & 0 & 0 \\
10 & 490 & 0.97 & 116 \\
11 & 340 & 0.97 & 81 \\
12 & $15{ }^{\prime} 300$ & 0.98 & 0 \\
13 & 0 & 0 & 0 \\
14 & 215 & 0.97 & 51 \\
\hline
\end{tabular}

\section{B. EVs input data}

The number of EVs per node is reported in Table I. It is estimated considering approx. 1.4 EVs per household ${ }^{2}$, where the number of households is estimated by dividing the nominal nodal power by the single-phase household contractual power (6 kVA). This results in total $878 \mathrm{EVs}$ for the whole grid. These values are aligned with the existing technical literature: the work in [15] considers $1.3 \mathrm{EVs}$ per household, with number of household per node estimated as the ratio between the nominal power of each residential node and the contractual power of a household (6 kVA); work [16] considers 2'340 EVs for a 5 MVA MV residential grid, denoting that more intense grid loading situations due to EVs may exist.

Arrival times, departure times and initial SOCs of EVs are sampled from the following distributions: generalized extreme

\footnotetext{
${ }^{2}$ Except for nodes 1 and 12, which have no EVs to reduce the number of optimization variables. This approximation is deemed reasonable because these nodes are near upper grid's connection, so less critical for grid constraints violations.
} 

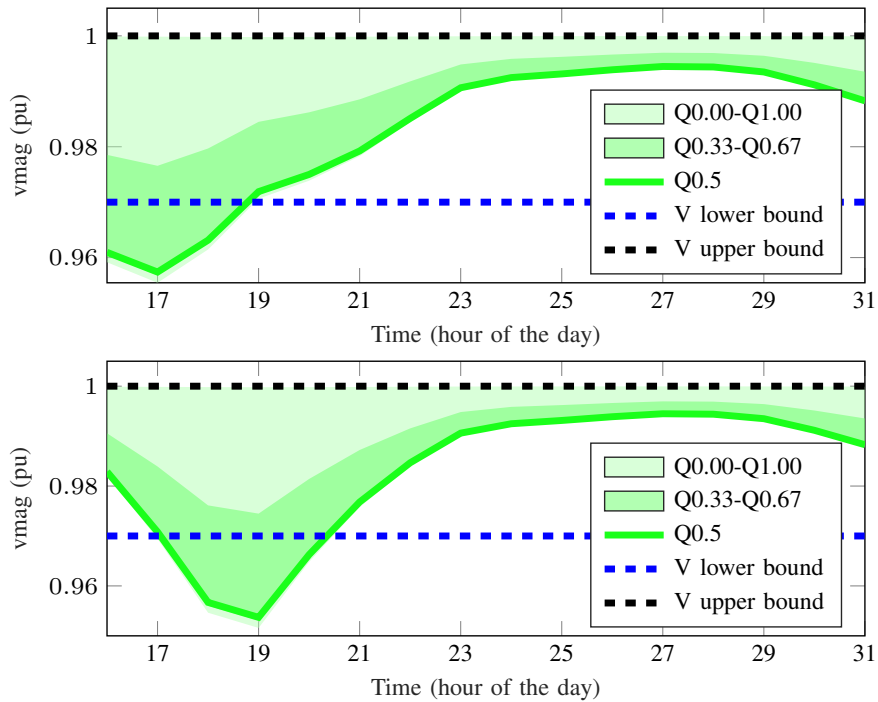

Fig. 2. Case 1: nodal voltage magnitudes over time with arrival time at $16 \mathrm{~h}$ for all vehicles (top panel), and with distributed arrival time (bottom panel). The dashed lines is the lower voltage limit. The shaded bands denote different quantile intervals across the nodes.

value inverse ( shape -0.06 , scale 0.85 , location 17.3 ), Weibull (scale 7.67, shape 21.83) and Gaussian (mean 0.49, standard deviation 0.04) distributions respectively as used in [17], [18]. The energy capacity of the EV batteries, and the chargers' rated power, efficiency, and power factor are assumed constant across their population and are $3.6 \mathrm{kVA}, 0.9$ and 1 , respectively. The target SOC levels are also uniform across the population and assumed to be $100 \%$.

\section{RESULTS AND Discussions}

Five cases are compared. They are:

- Case 1: uncoordinated charging (described in IV-C);

- Case 2 and Case 3: smart charging, without and with reactive power support, respectively (described in IV-B);

- Case 4 and Case 5: V2G, without and with reactive power support, respectively (described in IV-A).

Fig. 2 shows the nodal voltage magnitudes for case 1 . It compares the cases with simultaneous (at time 16h, top panel) and distributed arrival of the EVs (as described in V-B, bottom panel). The shades of color denote the quantile of the voltage magnitude across the nodes. In both cases, the voltage magnitudes falls below the minimum level because the uncoordinated charging does not take into account grid constraints.

In general, it can be expected that distributed arrivals of EVs induces a natural smoothing of the total charging demand because EVs arrive at different time, thus possibly "desynchronizing" their charging process. Indeed, Fig. 2 shows that, between $16 \mathrm{~h}$ and $17 \mathrm{~h}$, there are no voltage violations in the bottom plot, whereas there are in the upper-panel plot. However, in the evening hours, violations are more severe in the bottom panel than in the upper panel (as denoted by the lower voltage magnitude nadir) because the EV charging
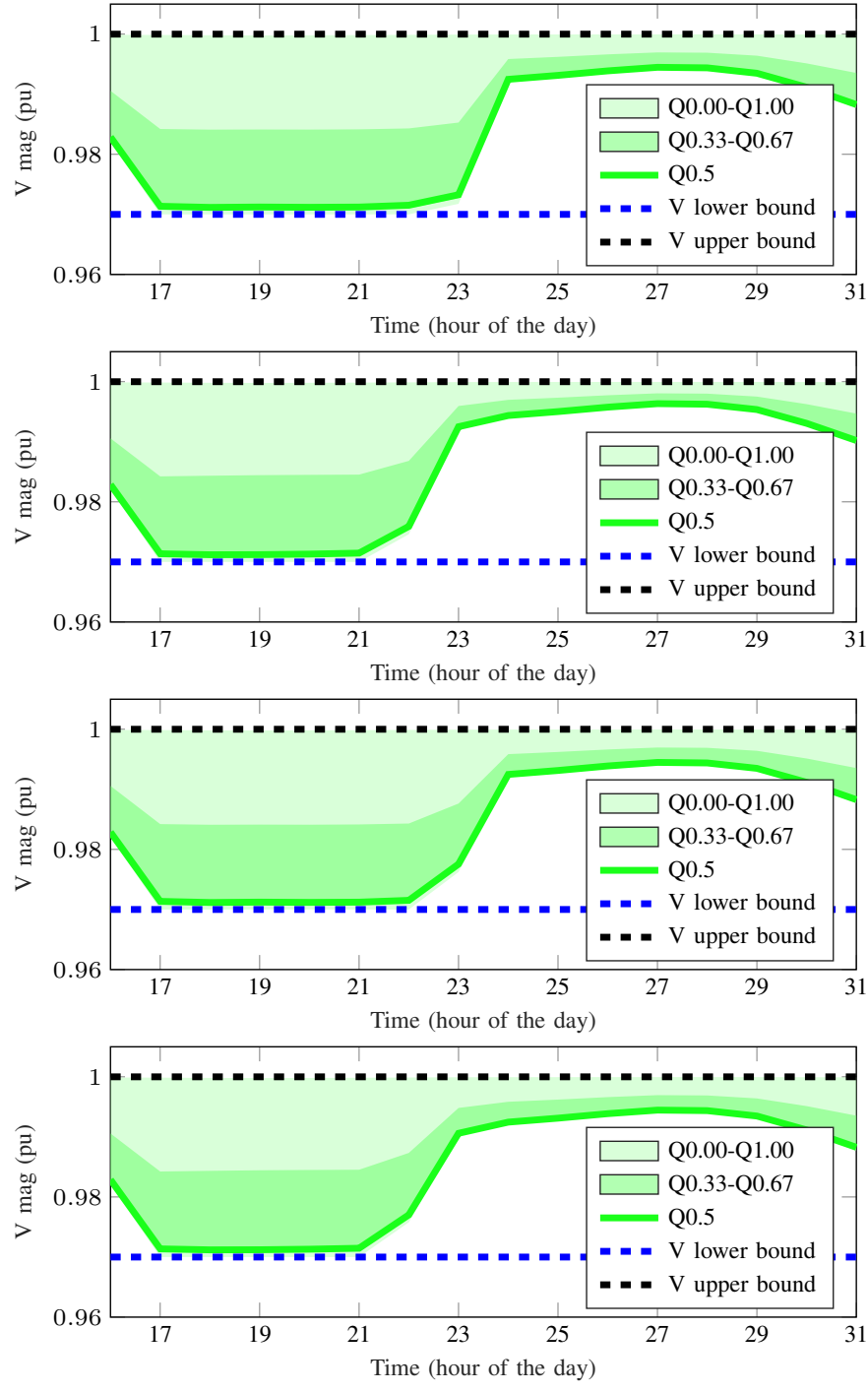

Fig. 3. Nodal voltage magnitudes over time for Case 2 (first panel from the top), Case 3 (second panel), Case 4 (third panel) and Case 5 (fourth panel). The dashed lines is the lower voltage limit. The shaded bands denote different quantile intervals across the nodes.

demand overlaps with the peak hours of conventional demand (reported in [11]). The rest of this analysis is performed considering the case with arrival times distributed in time.

Fig. 3 shows the voltage levels for Case 2 - 5. In all the cases, the voltage magnitudes are respected thanks to implementing grid constraints in the scheduling problem. Differences among the voltage profiles are due to handling the active and reactive power differently, as discussed next. Current constraints are also respected and omitted for a reason of space.

Fig. 4 shows the mean SOC across the EV population over time. Uncoordinated charging (Case 1) achieves the quickest recharging time. However, this comes at the price of violating grid constraints, as shown earlier. Smart charging and V2G (Case 2 and Case 4) achieves the slowest recharging time. This denotes that V2G does not conduce, in this case 


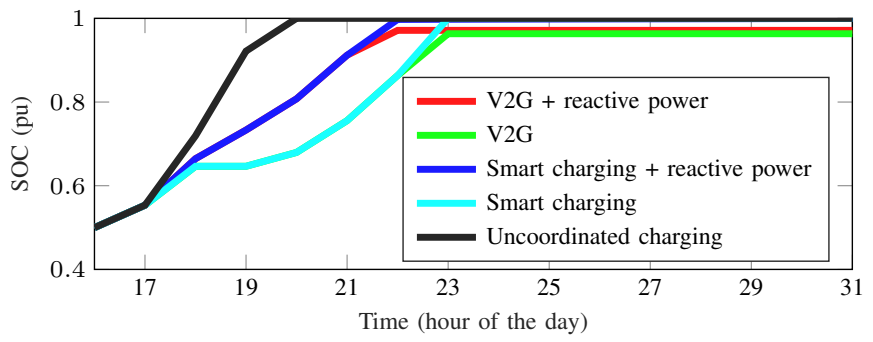

Fig. 4. Average SOC across the EVs population for different charging policies.

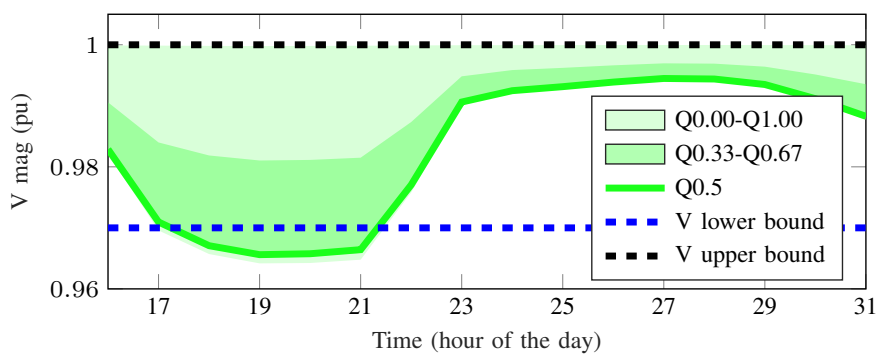

Fig. 5. Voltage magnitude computed by assuming the same active power demand as Case 3 but reactive power contribution from the EVs forced to zero.

to improve system performance. The difference between the steady-state SOC values of smart charging and $\mathrm{V} 2 \mathrm{G}$ is due to the modelling relaxation of the SOC. In particular, the cost function $J(\cdot)$ adopted in the $\mathrm{V} 2 \mathrm{G}$ problem trades off achieving the target SOC with charging power, determining a steady-state SOC error. This can be reduced by decreasing the value of $k$ in (11). The best-performing schedulers are smart charging and $\mathrm{V} 2 \mathrm{G}$ with reactive power support (Case 3 and Case 5). In this case too, V2G does not help to shorten charging times. To show the impact of reactive power support on the grid performance, Fig. 5 shows the voltage magnitude obtained from a (linearized) load flow that features the same active power demand of Case 3 but with the reactive power contributions from the chargers forced to zero. It is visible that voltage constraints are violated, showing that reactive power here plays an important role in keeping acceptable voltage levels in the MV feeders. The same results and conclusions were found by changing the target SOC levels to lower values (90\% and $80 \%)$.

\section{CONCLUSIONS}

This work compared the performance of uncoordinated charging, smart charging, V2G, and reactive power support from the chargers for EVs. The problem formulation of all the schedulers is derived from a common (convex) optimization problem accounting for (linearized) grid constraint, charging/discharging efficiency, and 4-quadrant (and 2-quadrant unidirectional) chargers, allowing for an efficient comparison among them. The performance of the schedulers is investigated considering the CIGRE system for MV grids with $878 \mathrm{EVs}$ distributed at the various nodes according to the nominal nodal power. The results show that: 1) uncoordinated charging determines violations of grid constraints; 2) smart charging is effective to reduce congestions; 3 ) the $\mathrm{V} 2 \mathrm{G}$ feature is never activated; and 4) reactive power support from the chargers achieves to reduce voltage congestions, ultimately leading to charge more EVs and shorten the global charging time.

\section{REFERENCES}

[1] "National institute of statistics and economic studies (INSEE)," April 2019.

[2] "EU 2030 climate \& energy framework," https://bit.ly/2P7PI0N, accessed: 2020-07.

[3] IRENA, "Innovation outlook: Smart charging for electric vehicles," International Renewable Energy Agency, Abu Dhabi, Tech. Rep. MSUCSE-06-2, 2019.

[4] M. S. ElNozahy and M. M. A. Salama, "A comprehensive study of the impacts of phevs on residential distribution networks," IEEE Transactions on Sustainable Energy, vol. 5, no. 1, pp. 332-342, 2014.

[5] J. A. P. Lopes, F. J. Soares, and P. M. R. Almeida, "Integration of electric vehicles in the electric power system," Proceedings of the IEEE, vol. 99, no. 1, pp. 168-183, 2011.

[6] M. López, S. Martín, J. Aguado, and S. de la Torre, "V2g strategies for congestion management in microgrids with high penetration of electric vehicles," Electric Power Systems Research, vol. 104, 2013.

[7] A. Zecchino, A. M. Prostejovsky, C. Ziras, and M. Marinelli, "Largescale provision of frequency control via $\mathrm{v} 2 \mathrm{~g}$ : The bornholm power system case," Electric Power Systems Research, vol. 170, 2019.

[8] K. Knezović, A. Soroudi, A. Keane, and M. Marinelli, "Robust multi-objective pq scheduling for electric vehicles in flexible unbalanced distribution grids," IET Generation, Transmission \& Distribution, vol. 11, no. 16, pp. 4031-4040, 2017. [Online]. Available: https://ietresearch.onlinelibrary.wiley.com/doi/abs/10.1049/ietgtd.2017.0309

[9] S. Paudyal, O. Ceylan, B. P. Bhattarai, and K. S. Myers, "Optimal coordinated ev charging with reactive power support in constrained distribution grids," in 2017 IEEE Power Energy Society General Meeting, 2017, pp. 1-5.

[10] K. Christakou, J. LeBoudec, M. Paolone, and D. Tomozei, "Efficient computation of sensitivity coefficients of node voltages and line currents in unbalanced radial electrical distribution networks," IEEE Transactions on Smart Grid, vol. 4, no. 2, pp. 741-750, 2013.

[11] CIGRE' Task Force C6.04.02, "Benchmark systems for network integration of renewable and distributed energy resources,' Cigre' International Council on large electric systems, Tech. Rep., July 2009.

[12] R. Gupta, F. Sossan, and M. Paolone, "Performance assessment of linearized opf-based distributed real-time predictive control," in 2019 IEEE Milan PowerTech, 2019, pp. 1-6.

[13] F. Sossan, B. Mukherjee, and Z. Hu, "Impact of the charging demand of electric vehicles on distribution grids: a comparison between autonomous and non-autonomous driving," in 2020 Fifteenth International Conference on Ecological Vehicles and Renewable Energies (EVER), 2020, pp. 1-6.

[14] E. Stai, F. Sossan, E. Namor, J.-Y. Le Boudec, and M. Paolone, "A receding horizon control approach for re-dispatching stochastic heterogeneous resources accounting for grid and battery losses," Electric Power Systems Research, vol. 185, p. 106340, 2020.

[15] S. Johansson, J. Persson, S. Lazarou, and A. Theocharis, "Investigation of the impact of large-scale integration of electric vehicles for a swedish distribution network," Energies, vol. 12, no. 24, 2019. [Online]. Available: https://www.mdpi.com/1996-1073/12/24/4717

[16] N. Leemput, F. Geth, J. Van Roy, P. Olivella-Rosell, J. Driesen, and A. Sumper, "Mv and $\mathrm{lv}$ residential grid impact of combined slow and fast charging of electric vehicles," Energies, vol. 8, no. 3, 2015.

[17] P. B. Andersen, "Intelligent electric vehicle integration-domain interfaces and supporting informatics," Ph.D. dissertation, Technical University of Denmark (DTU), 2013.

[18] "Test-an-EV project: Electrical Vehicle (EV) Data," http://mclabprojects.di.uniroma1.it/smarthgnew/Test-an-EV/?EVcode $=$ EV8, accessed: 2020-02 